\title{
Difficult dual-stage transcutaneous multiple lead extraction with loss of external silicone tube of broken lead
}

\author{
Andrzej Kutarski ${ }^{1}$, Michał Chudzik ${ }^{2}$, Andrzej Tomaszewski ${ }^{1}$, Radosław Pietura ${ }^{3}$, \\ Andrzej Oszczygiel ${ }^{2}$, Marek Czajkowski ${ }^{4}$, Jerzy Krzysztof Wranicz ${ }^{2}$ \\ ${ }^{1}$ Department of Cardiology, Medical University of Lublin, Poland \\ ${ }^{2}$ Department of Electrocardiology, First Chair of Cardiology and Cardiosurgery, \\ Medical University of Lodz, Poland \\ ${ }^{3}$ Department of Interventional Radiology and Neuroradiology, Medical University of Lublin, Poland \\ ${ }^{4}$ Department of Cardiosurgery, Medical University of Lublin, Poland
}

\begin{abstract}
The extraction of three implanted (18-, 16-, and ten year-old) damaged nonfunctional leads was complicated by a lead breaking and losing its external silicone tube. The missing part of the lead was invisible on X-ray, but was visible in ECHO as a thin, corded, very mobile limp structure without metallic reflection. Incomplete lead extraction did not cease laboratory symptoms of infection. The lost silicone tube was grasped and removed via femoral approach during the subsequent transesophageal echocardiography (TEE)-guided procedure. The presented case indicates that the criterion of full radiological success is not always correct, exposes the utility of ECHO techniques for X-ray-invisible broken lead fragments, and indicates the possibility of success for such TEE-guided procedures. (Cardiol J 2013; 20, 1: 94-99)
\end{abstract}

Key words: lead extraction, loss of silicone tube, TEE during lead extraction

\section{Introduction}

Infection in an implanted system is a categorical indication for its removal [1]. However, Heart Rhythm Society guidelines state that abandoning superfluous leads, even in young patients (progressive lead fixation by connecting tissue scar along its course) increases difficulties and the risks associated with its extraction in the future [2-4]. The risk of late extraction-related problems may be increased by previous attempts. Therefore, removal is indicated. The presented case illustrates a rare complication of damaged lead extraction in the shape of a lost long external silicone tube. It shows that 'full radiological success' is not always a suit- able description, and how ECHO might play a role in the solution of the problem.

\section{Case report}

A 69 year-old female with permanent atrial fibrillation (AF) and VVI pacemaker had a passive fixation lead (Tesla LSK 75) implanted 19 years previously via the left jugular vein. Three years later, an increase in pacing threshold led to the implantation of a new passive fixation endocardial lead (Biotronik 60 UP SN) via the right cephalic vein. Six years after that, a decrease in lead impedance with pacing and sensing disturbances, suggesting isolation damage, appeared. When its removal by

Address for correspondence: Andrzej Kutarski, MD, PhD, Department of Cardiology, Medical University of Lublin, ul. Jaczewskiego 8, 20-954 Lublin, Poland, tel./fax: +48 8172441 51, e-mail: a_kutarski@yahoo.com

Received: 11.11.2010

Accepted: 10.01.2011 


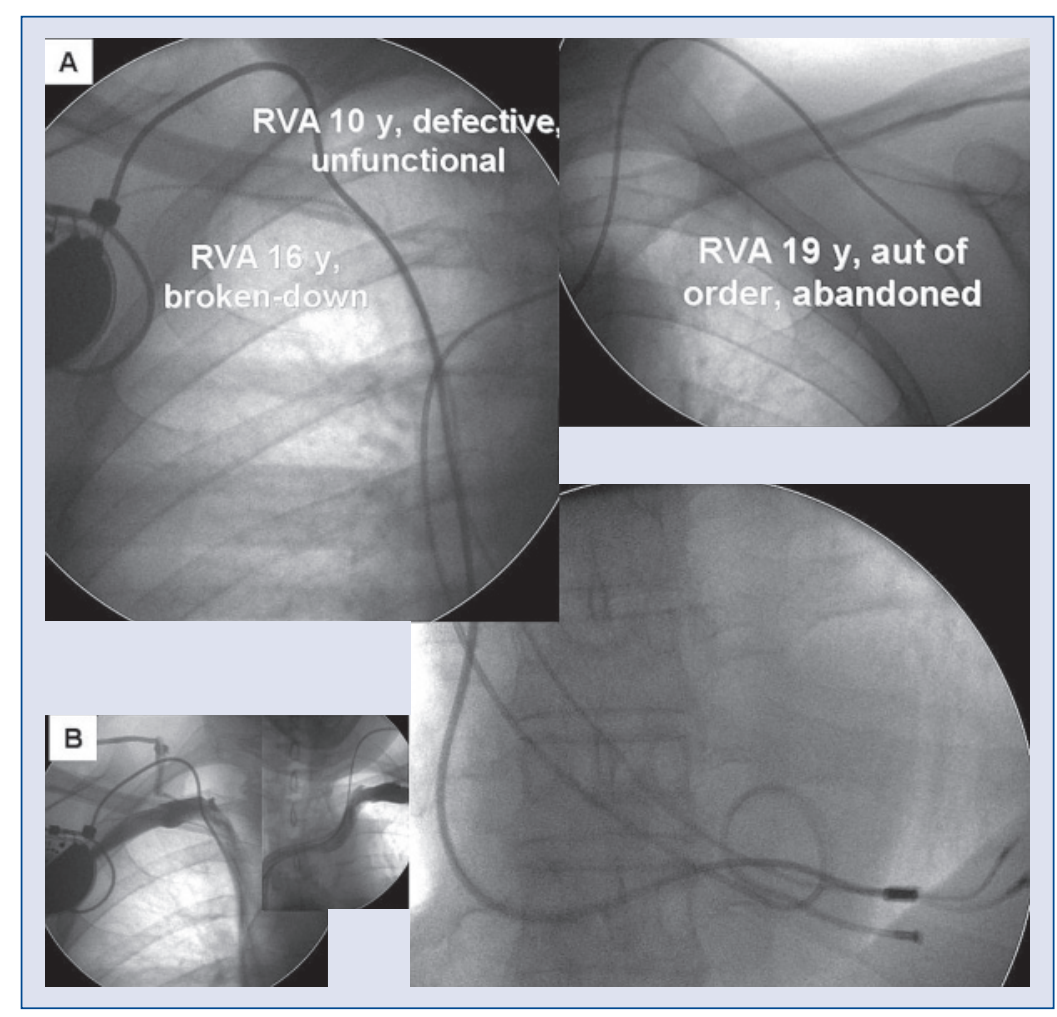

Figure 1. A. X-ray picture before lead extraction. Pandiculated V lead visible; B. Venography; RVA — right ventricular apex.

simple traction was attempted, it stretched and ruptured. The lead extraction failed and the proximal part of the lead was cut. Another lead was introduced (Biotronik TIR $60 \mathrm{BP}$ ) into the right jugular vein to gain access to the right ventricle (RV).

Unfortunately, after a few years, low impedance with very low R-wave amplitude with stimulating muscle, occasional oversensing, and pacing inhibition occurred in this lead as well. Thus, implantation of a new one, the subsequent endocardial lead, seemed necessary. Because of her having three old non-functional leads, with abnormal lead loop in the ventricle, the patient was referred to the Polish Reference Centre for the extraction of the abandoned leads (Fig. 1).

On admission, the patient was without fever, but showed ESR of $65 \mathrm{~mm} / \mathrm{h}$, WBC of $8,400 \mathrm{cell} / \mathrm{mL}$, CRP of $0.920 \mathrm{mg} / \mathrm{L}$, and d-dimer of $903.0 \mu \mathrm{g} / \mathrm{L}$. On ECHO examination, normal heart diameters $(\mathrm{LA}=4.0 \mathrm{~cm})$ with correct left ventricular ejection fraction $=62 \%$ as well as mitral and tricuspid valve regurgitation, were observed. Additionally, a conglomerate in RV with dimensions of $1.3 \times 1.2 \mathrm{~cm}$ was assessed. It was impossible to exclude an inflammatory system-related process.

The oldest lead, a 19 year-old lead implanted via the left jugular vein, was extracted first, using a mechanical system: polypropylene telescopic green Byrd dilators (Cook), but its tip became lodged in strong connecting tissue scar in the RV apex, and only partial radiological success was obtained.

The second one, a 10 year-old lead implanted via the right jugular vein, was extracted unbroken, demonstrating full radiological success using the same technique.

The final one, an abandoned, stretched and ruptured 16 year-old lead implanted via the right cephalic vein was extracted. It was impossible to obtain surgical access for the proximal ending of this lead, with the lead being at vein-entry side and using a femoral approach, and so the following attempt was made. The extracted lead was looped by a guidewire introduced via a pigtail catheter, and the distal part of the guidewire was grabbed by a Dotter basket (Fig. 2).

Manual traction was applied to the guidewire, and simultaneously to the basket catheter, and this broke the connection of the lead with its tip. Unfortunately, fixation to the occluded vein wall tissue scar of the connecting tissue surrounding the proximal damaged and long drawn-out lead ending, was found to be more resistant than the welded joint of the metal conductor with the tip of the extracted 


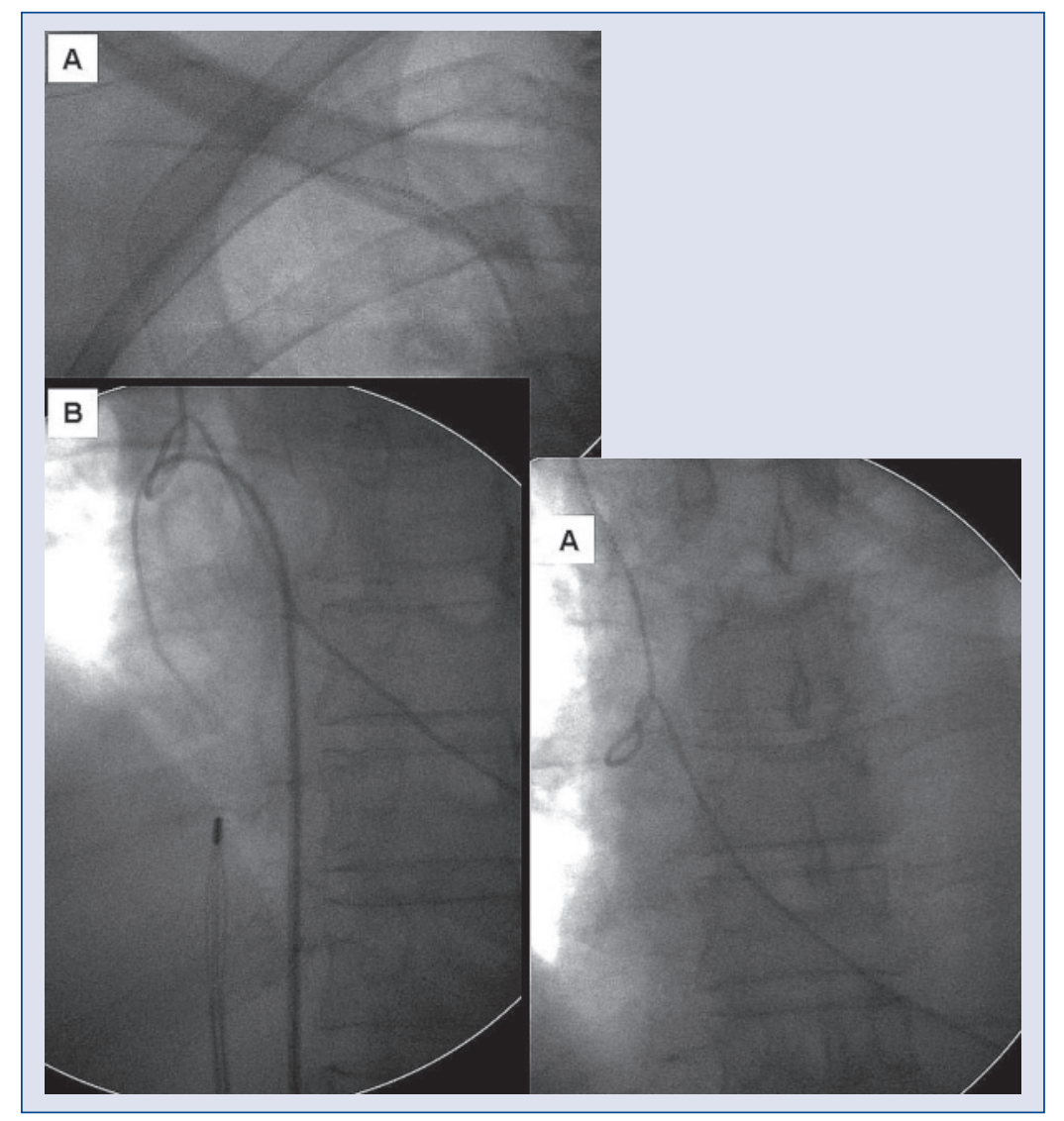

Figure 2. Extraction of the final lead. A. Both-side jugular vein implanted leads already extracted; remaining one destroyed; B. Looping extracted lead using pig-tail catheter, angiographic guidewire, and Dotter basket catheter.

lead. The connection was broken and one free ending of the lead, although a distal one, was obtained and was firmly grasped with a Dotter basket catheter. The tip (as with the second one) remained in the RV endocardium. Afterwards, $13 \mathrm{~F}$ sheath of the Femoral Work Station (Cook) with oblique tip (manually cut) was introduced. By rotating the catheter (in the same way as with the Byrd dilatators), we managed to liberate the proximal (subclavian) ending of the lead and removed it successfully, but without its silicone tube. At the moment of grasping the long-drawn-out lead, we could not evaluate what we caught in the Dotter basket (Figs. 3, 4).

Post-operative transesophageal echocardiography (TEE) performed with SONOS 5500 (Philips, Andover, MA, USA) revealed an unusual striped, thin, corded, very limp structure without metallic reflection. Incomplete lead extraction did not bring to an end the laboratory symptoms of infection, because leucocytosis with high levels of CRP was noted (Fig. 5).

Therefore, we decided to try to remove this 'invisible on X-ray' structure mainly using TEE to navigate the tools introduced through the femoral vein. Several dozen opening and closing basket catheters inside the right atrium, superior cava vein, and inferior cava vein were ineffective, but one out of the ten opening and closing lasso 35-mm catheter allowed us to catch something invisible, and pulling it changed the TEE picture. Finally, we removed the lasso catheter along with the lost $21-\mathrm{cm}-$ -long silicone tube, which was connected with the disappearance of the thin, corded, very limp, and mobile structure. The patient, after both the procedures, recovered quickly, with normalization of the laboratory markers of inflammatory process. TEE did not demonstrate abnormalities in the heart or available venous system (Fig. 6).

\section{Discussion}

Extraction of endocardial leads can cause complications connected to rupture, and the distal fragment can remain within the RV or venous system [5-7]. In our patient, the connecting tissue surrounding the proximal lead ending was very strong, and as with the first one, it was the distal part of the lead that was obtained, which was the cause of 


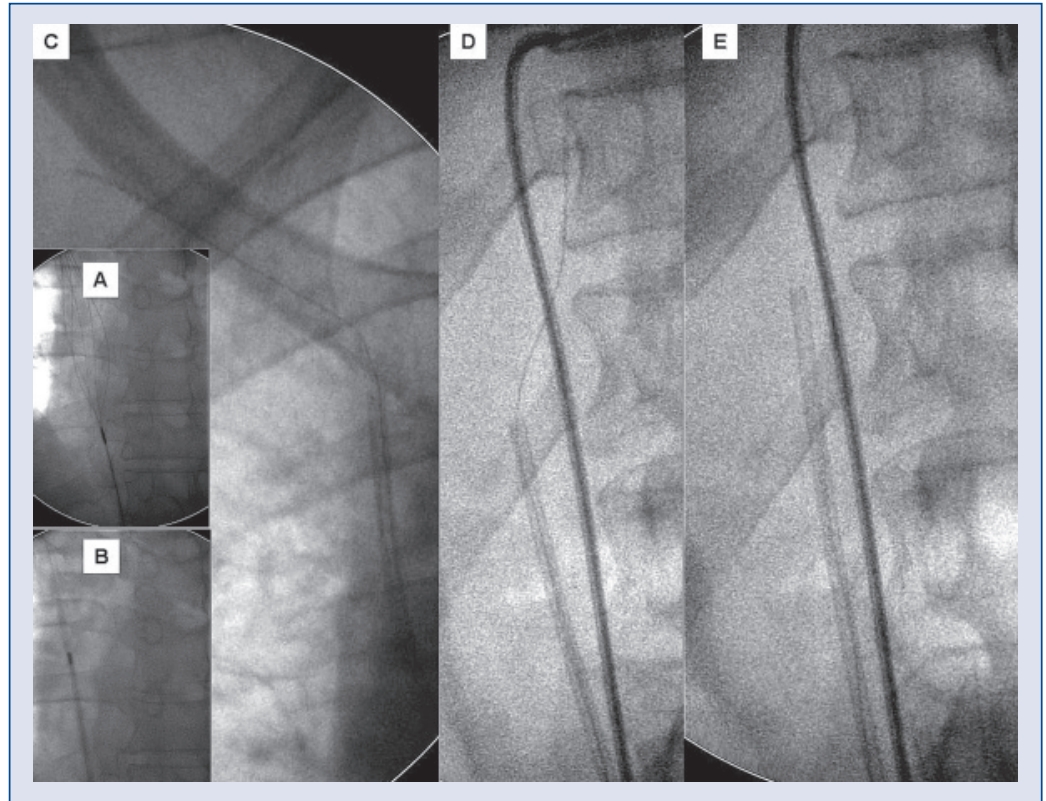

Figure 3. A, B. Distal ending of lead became free (without tip) and was grasped with Dotter catheter; C, D, E. Proximal part of lead liberation from the connecting tissue scars with $13 \mathrm{~F}$ teflon catheter with oblique tip (manually cut).

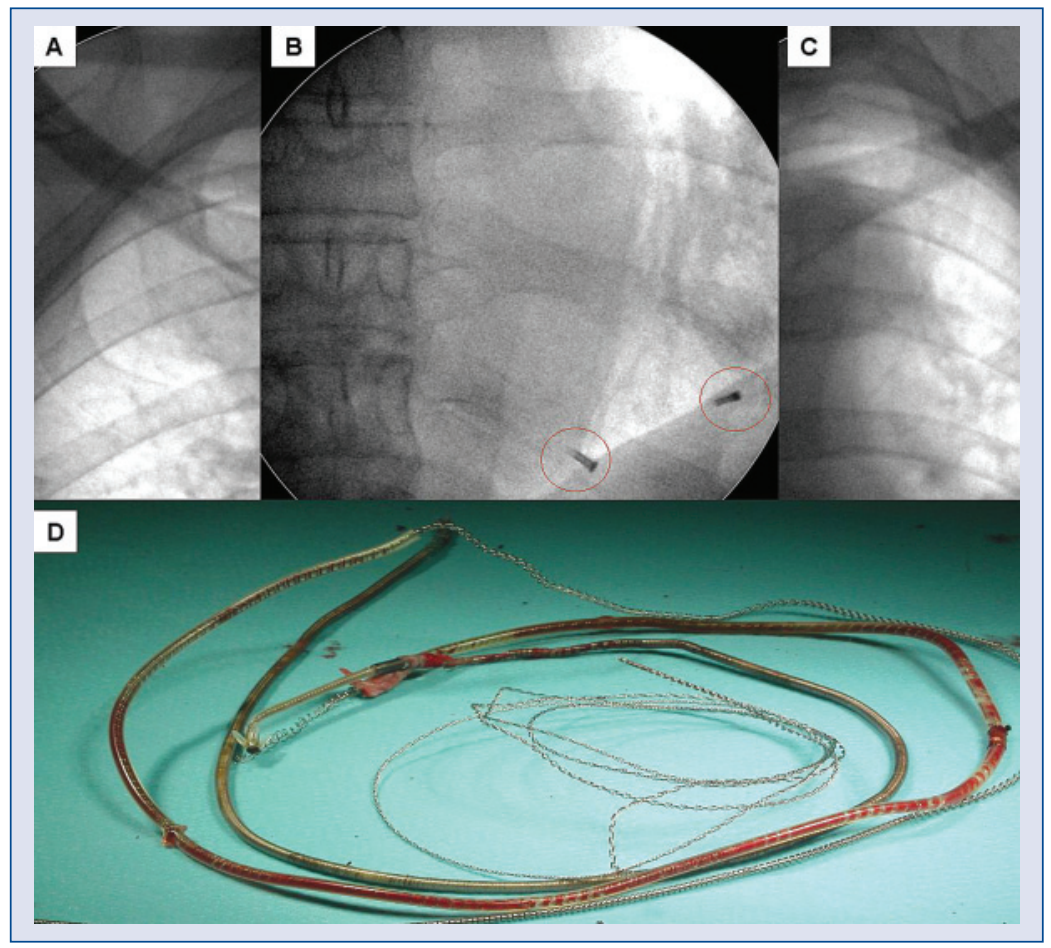

Figure 4A-D. Landscape after the battle; all leads extracted, but two tips of the leads remained in right ventricular endocardium. From the third (UP) lead, only the spiral metal conductor was extracted. 


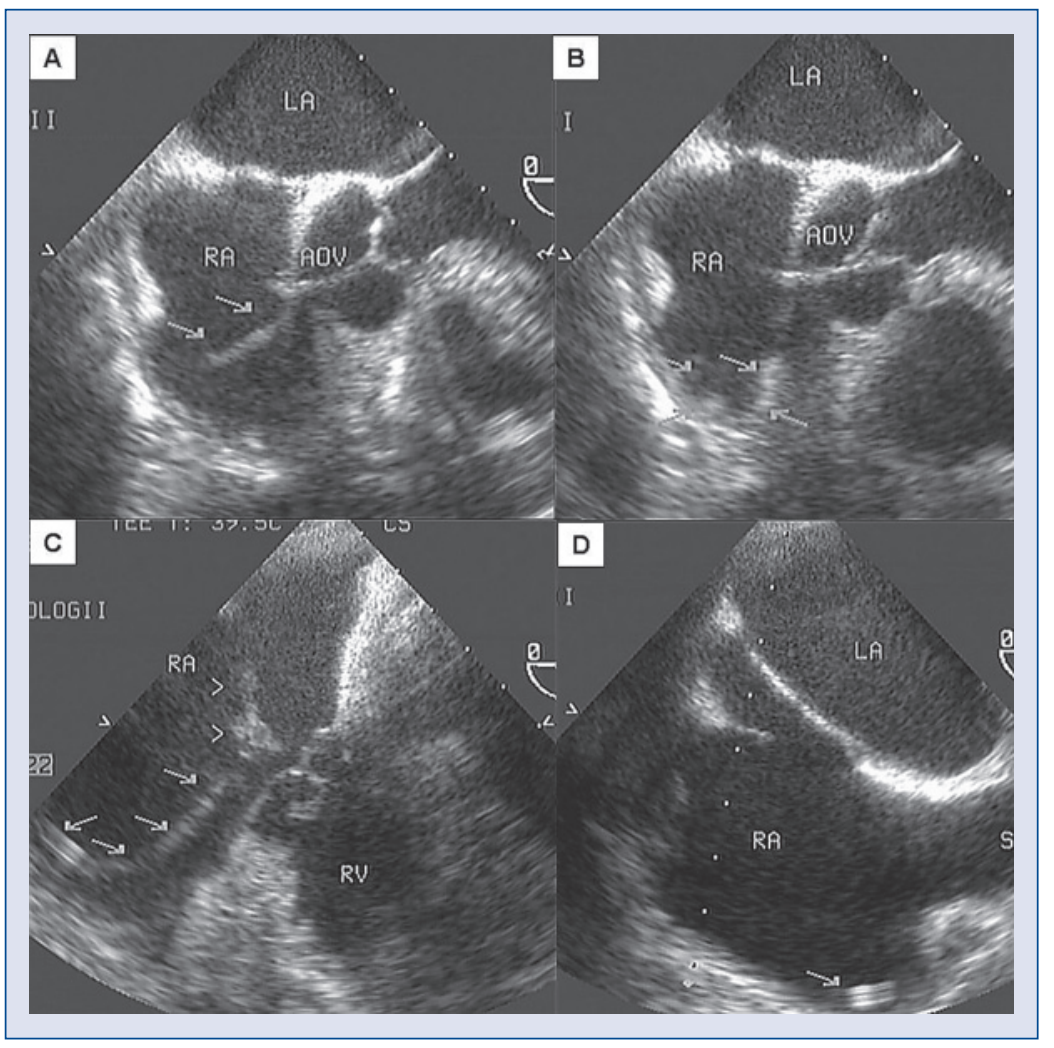

Figure 5. A. Transesophageal echocardiography after the procedure; arrows: in right atrium (RA), highly mobile structure with lead appearance; B. Transesophageal echocardiography; arrows: extensive movement of this structure $(A)$ in the same second; $C$. Transesophageal echocardiography; long arrows: long linear structure in RA, near RA wall connected to RA wall (appendage); short arrows: thickening of this structure with fibrotic tissue (confirmed after extraction); D. Transesophageal echocardiography; arrow: in this view, a small structure in RA appendage only is seen; LA — left atrium; RV — right ventricle; AOV — aortic valve.

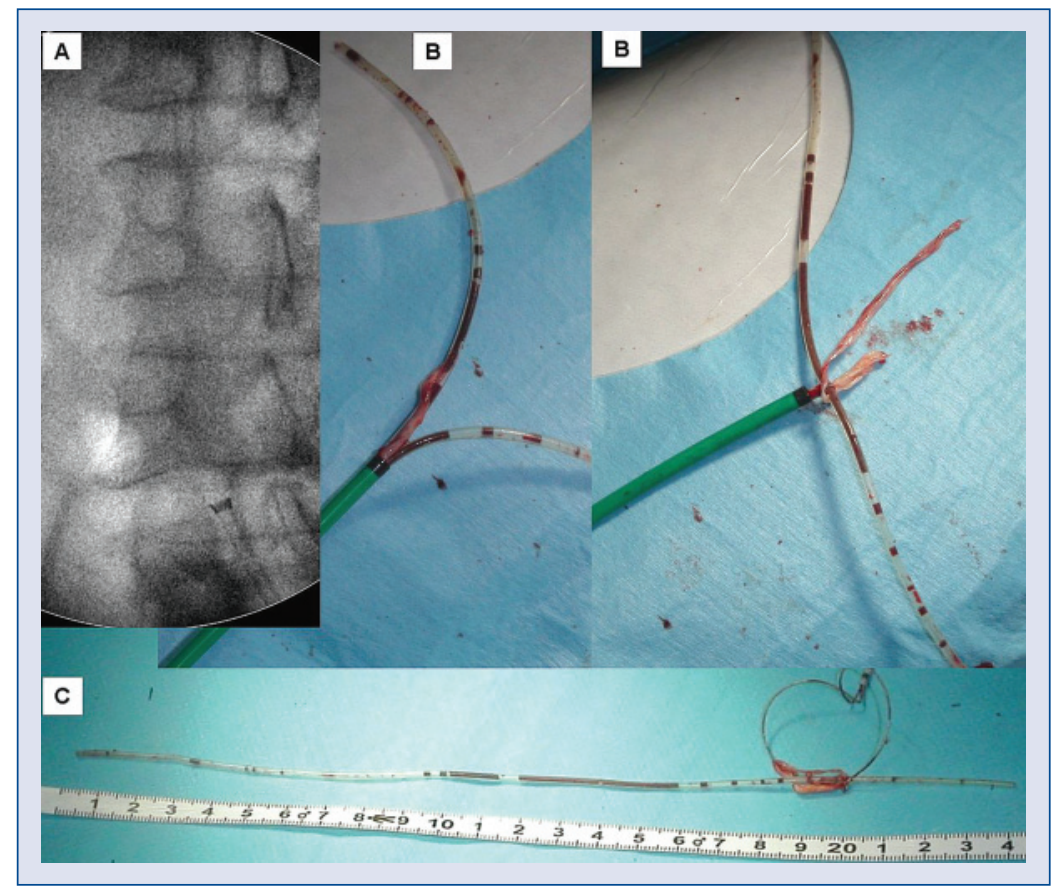

Figure 6. Silicone tube removal. A. Something invisible retracted with lasso catheter into the sheath; B, C. Removed during previous silicone tube procedure. 
atypical extraction with surprising result. Several studies have demonstrated the usefulness of ECHO examination in the diagnosis of infection and complications during lead extraction [8-10]. However, to the best of our knowledge, ours is the first case in which TEE examination alone revealed an 'invisible structure' which was successfully removed through the joint efforts of an electrophysiolgist, interventional radiologist, and cardiologist with ECHO examination experience.

This case proves that patients with increasing complications where there is a need for 'troubleshooting' will be referred to our hospitals. So, we must always provide the best high-volume medical centers to treat their complications. Such complications pose real challenges and highlight the need for experienced interventional radiologists and cardiologists with great ECHO examination experience.

\section{Conclusions}

The presented case is of a rare but serious leadextraction complication owing to the loss of a lead silicone tube. Complete radiological success is not the only definition of success. Our case shows that ECHO techniques can be used to detect X-ray-invisible broken lead fragments, and indicates the possibility of success of a TEE-guided procedure.

Conflict of interest: none declared

\section{References}

1. Wilkoff BL, Love CJ, Byrd CL et al. Heart Rhythm Society; American Heart Association. Transvenous lead extraction: Heart Rhythm Society expert consensus on facilities, training, indications, and patient management: This document was endorsed by the American Heart Association (AHA). Heart Rhythm, 2009; 6: 1085-1104.

2. Hamid S, Arujuna A, Ginks M et al. Pacemaker and defibrillator lead extraction: Predictors of mortality during follow-up. Pacing Clin Electrophysiol, 2010; 33: 209-216.

3. Hauser RG, Katsiyiannis WT, Gornick CC, Almquist AK, Kallinen LM. Deaths and cardiovascular injuries due to device-assisted implantable cardioverter-defibrillator and pacemaker lead extraction. Europace, 2010; 12: 395-401.

4. Venkataraman G, Hayes DL, Strickberger SA. Does the riskbenefit analysis favor the extraction of failed, sterile pacemaker and defibrillator leads? J Cardiovasc Electrophysiol, 2009; 20: 1413-1415.

5. Bongiorni MG, Soldati E, Zucchelli G et al. Transvenous removal of pacing and implantable cardiac defibrillating leads using single sheath mechanical dilatation and multiple venous approaches: High success rate and safety in more than 2,000 leads. Eur Heart J, 2008; 29: 2886-2893.

6. Scott PA, Chow W, Ellis E, Morgan JM, Roberts PR Extraction of pacemaker and implantable cardioverter defibrillator leads: A single-centre study of electrosurgical and laser extraction. Europace, 2009;. 11: 1501-1504.

7. Kennergren C, Bjurman C, Wiklund R, Gäbel J. A single-centre experience of over 1,000 lead extractions. Europace, 2009; 11: 612-617.

8. Grammes JA, Schulze CM, Al-Bataineh M et al. Percutaneous pacemaker and implantable cardioverter-defibrillator lead extraction in 100 patients with intracardiac vegetations defined by transesophageal echocardiogram. J Am Coll Cardiol, 2010; 55: 886-994.

9. Endo Y, O'Mara JE, Weiner S et al. Clinical utility of intraprocedural transesophageal echocardiography during transvenous lead extraction. J Am Soc Echocardiogr, 2008; 21: 861-867.

10. Rizzello V, Dello Russo A, Casella M, Biddau R. Residual fibrous tissue floating in the right atrium after percutaneous pacemaker lead extraction: An unusual complication early detected by intracardiac echocardiography. Int J Cardiol, 2008; 127: 67-68. 